\title{
Both radical prostatectomy following treatment with neoadjuvant LHRH agonist and estramustine and radiotherapy following treatment with neoadjuvant hormonal therapy achieved favorable oncological outcome in high-risk prostate cancer: a propensity-score matching analysis
}

Takuya Koie ${ }^{1}$, Chikara Ohyama ${ }^{*}$, Hayato Yamamoto ${ }^{1}$, Atsushi Imai , Shingo Hatakeyama', Takahiro Yoneyama', Yasuhiro Hashimoto', Tohru Yoneyama', Yuki Tobisawa', Masahiko Aoki ${ }^{2}$ and Yoshihiro Takai

\begin{abstract}
Background: To date, the different treatment modalities for high-risk prostate cancer (Pca) have not been compared in any sufficiently large-scale, prospective, randomized clinical trial. We used propensity-score matching analysis to compare the oncological outcomes of high-risk prostate cancer between patients treated with radical prostatectomy (RP) and those treated with radiation therapy (RT).

Methods: We studied 216 patients who received neoadjuvant therapy followed by RP (RP cohort) and 81 patients who received neoadjuvant androgen-deprivation therapy (ADT) followed by RT (RT cohort). The RP cohort received a luteinizing hormone-releasing hormone agonist and estramustine phosphate ( $280 \mathrm{mg} /$ day) for 6 months prior to RP. The RT cohort received ADT for at least 6 months prior to RT using a 3-dimensional conformal radiotherapy technique. The total radiation dose was 70 to 76 Gy administered at 2 Gy/fraction.

Results: Propensity-score matching identified 78 matched pairs of patients. The 3-year overall survival rates were $98.3 \%$ and $92.1 \%$ in the RP and RT groups, respectively $(P=0.156)$. The 3-year biochemical recurrence-free survival rates were $86.4 \%$ and $89.4 \%$ in the RP and RT groups, respectively $(P=0.878)$.

Conclusions: Our study findings may suggest almost identical cancer control of RP and RT with appropriate neoadjuvant therapy in high-risk Pca. Therefore, issues of health-related quality of life may have an important impact on decision making in treatment of high-risk Pca.
\end{abstract}

Keywords: Biochemical outcomes, Propensity-score matching, Prostate cancer, Radical prostatectomy, Radiation therapy

\section{Background}

Individuals with prostate-specific antigen (PSA) levels of $\geq 20 \mathrm{ng} / \mathrm{mL}$, Gleason scores of $\geq 8$, or clinical stage T2c/T3 tumors are defined as high-risk prostate cancer (Pca) patients [1]. Treatment options for high-risk Pca include external beam-radiation therapy (EBRT) with

\footnotetext{
* Correspondence: coyama@cc.hirosaki-u.ac.jp

'Department of Urology, Hirosaki University Graduate School of Medicine, 5

Zaifucho, Hirosaki 036-8562, Japan

Full list of author information is available at the end of the article
}

androgen-deprivation therapy (ADT); trimodal therapy with a combination of brachytherapy, EBRT, and ADT; and radical prostatectomy (RP) with neoadjuvant or adjuvant therapy. To date, no sufficiently large-scale, prospective, randomized clinical trials have compared the abovementioned treatment options. Thus, optimal management strategies for high-risk Pca patients have not been established. Previous studies comparing RP and EBRT were difficult to interpret because of biased treatment selection criteria, incomplete follow-up data, varied 
treatment protocols, and reliance on surrogate endpoints [2]. Above all, several studies comparing Pca treatment options have either overlooked medical comorbidities, because of lack of relevant information [3], or have attempted to control for measured comorbidities using statistical methods $[4,5]$.

Therefore, we aimed to evaluate the overall survival (OS) and the biochemical recurrence-free survival (BRFS) rates of high-risk Pca patients who underwent either RP or EBRT using propensity-score matching analyses to adjust for treatment selection bias.

\section{Methods}

\section{Patient selection}

We conducted a retrospective chart review of 329 consecutive high-risk Pca patients treated at our institution between July 2004 and July 2012. Thirty-two patients who underwent only RP were excluded. We selected 216 patients who received neoadjuvant therapy followed by RP (the RP cohort) and 81 patients who received neoadjuvant ADT followed by EBRT (the RT cohort). The study protocol and informed consent documents was reviewed and approved by the Hirosaki University institutional review board.

\section{Treatment}

A single pathologist reviewed the diagnostic biopsy specimens and surgical specimens. We have previously reported the active effect of luteinizing hormone-releasing hormone (LHRH) plus low-dose estramustine phosphate (EMP; LHRH + EMP) for high-risk Pca patients [6]. Patients in the RP cohort received LHRH (leuprolide (11.25 mg) or goserelin acetate $(10.8 \mathrm{mg})$ every 3 months) and EMP (280 mg/day) for 6 months prior to RP [6]. Retropubic RP was performed as previously described in detail [7]. All patients in the RP cohort underwent the same lymphadenectomy procedure, which included removal of the bilateral obturator lymph nodes.

RT patients received ADT (LHRH and an antiandrogen) for at least 6 months prior to receiving EBRT. All patients were treated using a 3-dimensional conformal radiotherapy (3D-CRT) technique. The clinical target volume included the entire prostate and the bases of the seminal vesicles. A safety margin of $10 \mathrm{~mm}$ was added in all directions except posteriorly, where a 6-mm margin was added, to create the planning target volume. The total radiation dose was 70 to 76 Gy delivered in 2 Gy/ fraction at 5 fractions/week.

\section{Follow-up evaluations}

All patients were followed up by assessing serum PSA and testosterone levels every 3 months for 5 years and every 6 months thereafter. Pretreatment serum PSA levels were measured within 1 month of RP or EBRT administration. No patient was lost to follow-up in this study.

For RP-treated patients, disease recurrence or PSA failure was defined as serum PSA levels exceeding $0.2 \mathrm{ng} / \mathrm{mL}$. If PSA levels did not decrease to less than $0.2 \mathrm{ng} / \mathrm{mL}$ after surgery, the date of RP was defined as the date of disease recurrence. For EBRT-treated patients, PSA failure was defined according to the 2006 consensus statement by the American Society of Therapeutic Radiation and Oncology [8]. PSA levels rising by $2 \mathrm{ng} / \mathrm{mL}$ or more above the nadir PSA levels is currently defined as biochemical failure after EBRT [8].

\section{Statistical analysis}

To reduce the effect of treatment selection bias and potential confounding factors, we performed propensityscore matching analysis [9]. Propensity scores were calculated for each patient using multivariate logistic regression analysis including the following covariates: age, pretreatment PSA levels, biopsy Gleason scores, and clinical tumor staging. Tumors were staged according to the 2002 Staging Manual by the American Joint Committee on Cancer Staging [10]. The Gleason scores for prostate biopsy cores and surgical specimens were determined according to the 2005 guidelines by the International Society of Urological Pathology [11]. The predicted values according to the regression model estimated the propensity of each patient for receiving RP or EBRT according to his/her baseline characteristics. The differences between the two groups were assessed by fitting a logistic regression model using treatment as the response variable and baseline characteristics as covariables. Data were analyzed using IBM SPSS Statistics 20 software (International Business Machines Corp., New York, USA). OS and BRFS rates were analyzed using the Kaplan- Meier estimator. The relationship between survival and subgroup classification was analyzed using the log-rank test. All $P$ values were two-sided and the significance level was set at $P<0.05$.

\section{Results}

\section{Patient characteristics}

Propensity-score matching identified 78 matched pairs of patients. Table 1 shows the pretreatment clinical characteristics of the two groups. No differences were noted in age, initial PSA levels, biopsy Gleason scores, or clinical $\mathrm{T}$ staging between the two groups. The median follow-up periods of the RP- and EBRT-treated patients were not significantly different.

In the RT cohort, the median duration of ADT prior to receiving RT was 12 months (interquartile range: 9 to 16 months). Fifty-four patients (69\%) received RT at a dose of $70 \mathrm{~Gy}$, and 24 patients (31\%) received RT at a dose of 74 Gy. 
Table 1 Pretreatment clinical characteristics categorized according to treatment administered to 156 patients with high-risk prostate cancer, adjusted for propensity scores

\begin{tabular}{llll}
\hline $\begin{array}{l}\text { Pretreatment } \\
\text { characteristics }\end{array}$ & $\begin{array}{l}\text { Radiation } \\
\text { therapy } \\
\mathbf{( N = 7 8 )}\end{array}$ & $\begin{array}{l}\text { Radical } \\
\text { prostatectomy } \\
(\mathbf{N}=\mathbf{7 8})\end{array}$ & $\mathbf{P}$ \\
\hline Age (year, median) & 73.5 & 71 & $0.0633^{\mathrm{a}}$ \\
\hline $\begin{array}{l}\text { Initial prostate-specific } \\
\text { antigen level } \\
\text { (ng/mL, median) }\end{array}$ & 21.42 & 20.00 & 0.3886 \\
\hline Clinical T stage & & & 0.8482 \\
\hline T1c & 19 & 22 & \\
\hline T2 & 23 & 19 & \\
\hline T3 & 36 & 37 & \\
\hline
\end{tabular}

Biopsy Gleason score

\begin{tabular}{lllll}
\hline$\leq 6$ & 5 & 4 & 0.9384 \\
\cline { 1 - 3 } & 24 & 23 & \\
\hline$\geq 8$ & 49 & 51 & 0.3338
\end{tabular}

(month, median)

${ }^{\mathrm{a}} P$ values indicate statistical significance.

Pathological outcomes in the radical prostatectomy cohort All patients in the RP cohort were evaluated for pathological response. Regarding the pathological T stage, 5\%, $59 \%$, and $36 \%$ of patients had pT0, pT2, and pT3 tumors, respectively. Seven patients (9\%) had positive surgical margins in the surgical specimens. None of the patients had received adjuvant therapy, including ADT or RT.

\section{Oncological outcomes}

The 3-year OS rates were $98.3 \%$ and $92.1 \%$ for the RP and RT cohorts, respectively ( $\mathrm{P}=0.156$; Figure 1$)$. At the time of analysis, 5 RT patients had died. The causes of death were prostate cancer, colorectal cancer, hepatocellular carcinoma, cerebral hemorrhage, and chronic heart failure. One patient from the RP cohort committed suicide. The 3-year BRFS rates were $86.4 \%$ and $89.4 \%$ in the $\mathrm{RP}$ and RT cohorts, respectively $(\mathrm{P}=0.878$; Figure 2$)$. At the time of analysis, PSA failure had occurred in 11 RT and $9 \mathrm{RP}$ patients. These patients did not show clinical recurrence except for 1 RT-treated patient. In the RP cohort, the 3-year BRFS rate was 89.9\% (95\% confidence interval (CI): 66.5 to 77.7$)$ in patients who achieved pathological T0/T2 status, and $78.1 \%$ (95\% CI: 45.4 to 73.9 ) in those with T3 status $(\mathrm{P}=0.018)$.

\section{Discussion}

To our knowledge, the efficacies of EBRT and RP in terms of biochemical outcomes, particularly in high-risk Pca patients, have not been compared in randomized controlled trials; therefore, reliance on observational data has become mandatory. A comparative analysis of studies involving prostate cancer treated with various modalities was conducted by the Prostate Cancer Results Study Group. The analysis, authored by Grimm et al., provides some insight into the relative effectiveness of surgery and RT for high-risk disease [12]. Combination therapies involving RT and brachytherapy plus or minus ADT appear superior to more localized treatments such as RP alone or RT alone [12]. In the present study, OS and PSA outcomes following $\mathrm{RP}$ or 3D-CRT were compared among high-risk Pca patients who were matched for pretreatment predictors.

We administered neoadjuvant LHRH + EMP followed by RP to high-risk Pca patients. Among high-risk Pca patients, reported rates of PSA-free survival after RP alone was 35 to $62 \%$ [13,14]. Furthermore, neoadjuvant hormone therapy before RP reduces the rate of positive surgical margins, potentially resulting in pathologic complete responses. However, neoadjuvant ADT has not been shown to be beneficial for patient outcomes, especially in terms of PSA-free survival, in randomized trials [15]. Long-term administration of low-dose EMP may have a positive impact on the PSA-free survival rate; the PSA-free survival rate was $86.4 \%$ in our study, which was higher than the values reported by several other clinical trial [13-15].

In EBRT-treated high-risk Pca patients, an RT dose of 70 Gy may be inadequate to eradicate the disease completely. Support for this hypothesis came from a randomized dose escalation trial (78 Gy versus 70 Gy) [16], in which a beneficial effect was noted for all patients in terms of the 5 -year BRFS rate (78\% versus $68 \% ; P=0.03)$, and particularly, in patients with a pretreatment PSA level of $>10$ to $20 \mathrm{ng} / \mathrm{mL}$ ( $72 \%$ versus $43 \% ; P=0.01$ ). Patients with locally advanced Pca were found to experience favorable survival outcomes in a prospective randomized clinical trial when ADT was added to EBRT [17].

In fact, a more favorable outcome may be achieved with neoadjuvant LHRH + EMP and RP or 3D-CRT and ADT than RP or 3D-CRT alone in high-risk Pca patients. Our study findings may suggest almost identical cancer control by RP and RT with appropriate neoadjuvant therapy in high-risk Pca. On the other hand, it is interesting to note that benefits in terms of BRFS were not observed after treatment completion in the two groups in the present study. This observation may be attributable to the differing definition of PSA failure between RP- and EBRT-treated patients. PSA must reach the nadir value in patients treated with EBRT, and this can take 1 to 2 years or occasionally longer [18]. Conversely, almost all RP-treated patients will achieve the nadir PSA value within 1 to 2 months or sooner after therapy. Therefore, clinically meaningful and reliable results require longer follow-up periods.

Finally, the current study was not performed as a noninferiority study to compare the efficacy of RP with the RT in patients with high-risk Pca. Our findings were limited by 


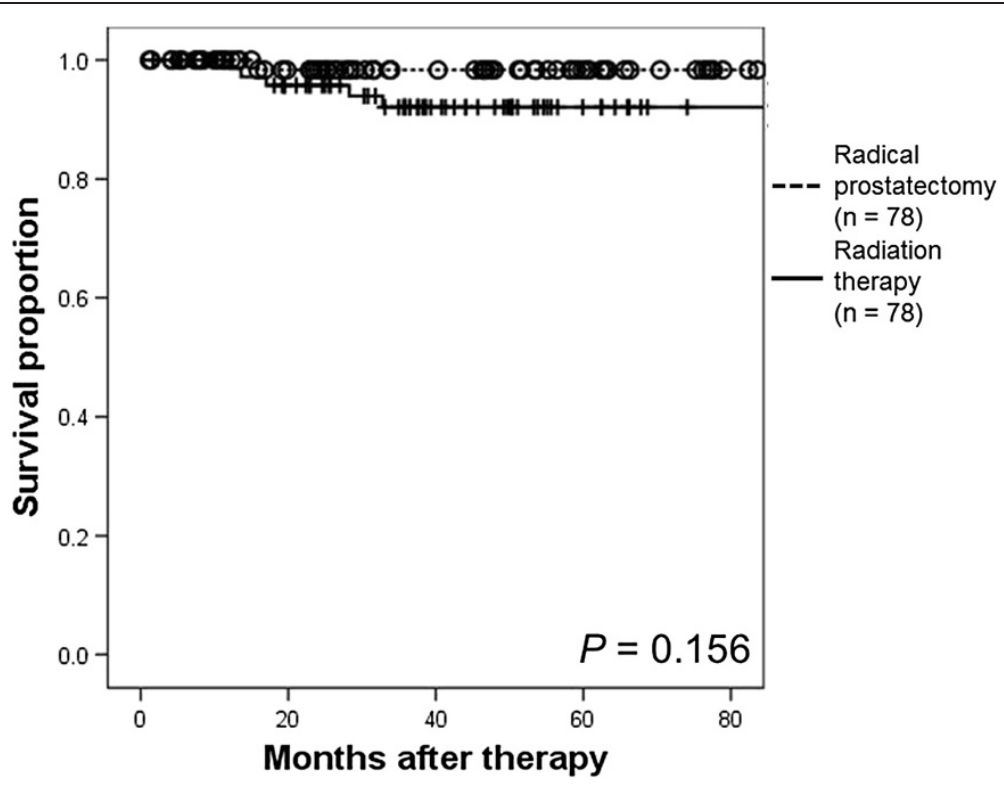

Figure 1 Kaplan- Meier estimate of overall survival (OS). The 3-year OS rate was $98.3 \%$ in the high-risk prostate cancer patients treated with neoadjuvant luteinizing hormone-releasing hormone agonist and estramustine phosphate followed by radical prostatectomy. The 3-year OS rate was $92.1 \%$ in patients treated with neoadjuvant androgen-deprivation therapy followed by radiation therapy $(P=0.156)$.

the retrospective nature of our study and the relatively small study sample size. The RP patients received neoadjuvant LHRH + EMP, and RT patients received neoadjuvant LHRH and antiandrogen. Propensity-score analysis is a method used to reduce bias in observational studies and matching was limited to available variables. Additionally, other factors such as quality of life, continence, and erectile function, which also affect treatment decisions, were not evaluated in our study. Therefore, issues of health-related quality of life may have an important impact on decision making of treatment in high-risk prostate cancer. However, our study results may assist in decision-making

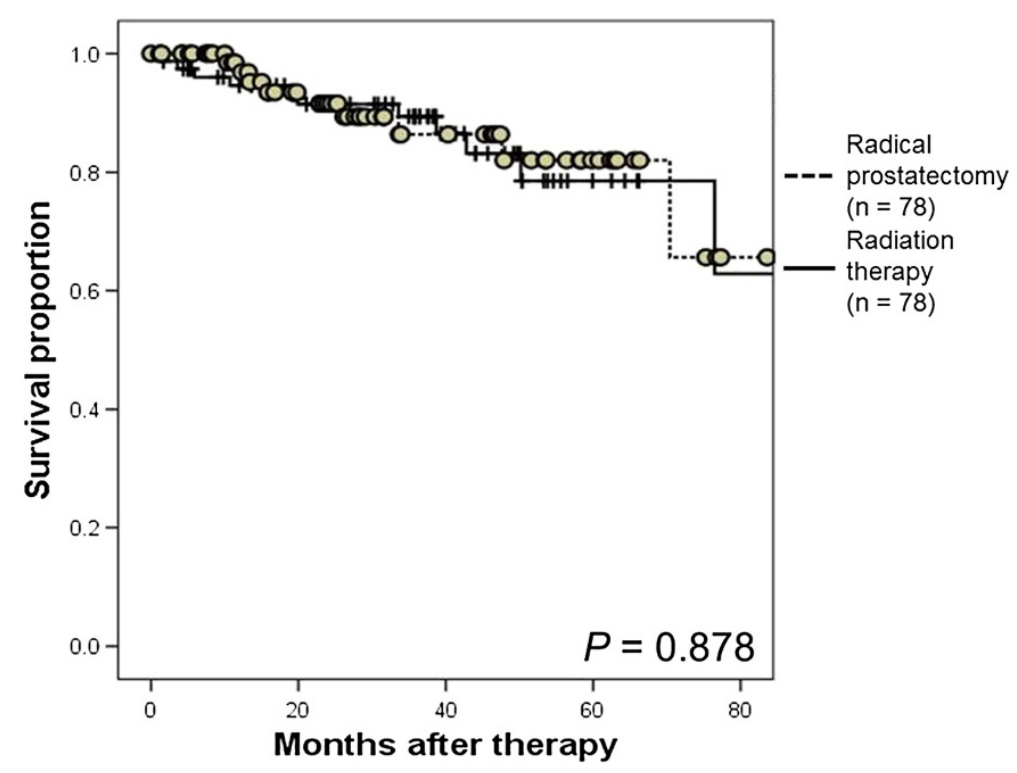

Figure 2 Kaplan- Meier estimate of biochemical recurrence-free survival (BRFS). The 3-year BRFS rates were $86.4 \%$ in the high-risk prostate cancer patients treated with neoadjuvant luteinizing hormone-releasing hormone agonist and estramustine phosphate followed by radical prostatectomy and $89.4 \%$ in those treated with neoadjuvant androgen-deprivation therapy followed by radiation therapy $(P=0.878)$. 
for managing high-risk Pca patients because prospective randomized clinical trial data are lacking. Future clinical trials are warranted.

\section{Conclusions}

Our study findings may suggest almost identical cancer control of RP and RT with appropriate neoadjuvant therapy in high-risk Pca. Therefore, issues of health-related quality of life may have an important impact on decision making in treatment of high-risk Pca.

\section{Abbreviations}

ADT: androgen-deprivation therapy; BRFS: biochemical recurrence-free survival; EBRT: external beam-radiation therapy; LHRH + EMP: luteinizing hormonereleasing hormone agonist and estramustine phosphate; OS: overall survival; Pca: prostate cancer; PSA: prostate-specific antigen; RP: radical prostatectomy; RT: radiation therapy; 3D-CRT: 3-dimensional conformal radiotherapy.

\section{Competing interests}

The authors declare that they have no competing interests.

\section{Authors' contributions}

TK wrote the manuscript. HY, Al, SH, TY and MA performed clinical follow-up examinations and contributed to the manuscript. $\mathrm{YH}$ reviewed the pathological specimens. YT, TY and YT contributed to manuscript drafting. CO was responsible for the concept, design, data interpretation, and critical revision of the manuscript. All authors read and approved the final version of the manuscript.

\section{Acknowledgements}

No funding was received for the present study.

\section{Author details}

'Department of Urology, Hirosaki University Graduate School of Medicine, 5 Zaifucho, Hirosaki 036-8562, Japan. ²Department of Radiology, Hirosaki University Graduate School of Medicine, 5 Zaifucho, Hirosaki 036-8562, Japan.

Received: 20 September 2013 Accepted: 15 April 2014

Published: 30 April 2014

\section{References}

1. Lester-Coll NH, Goldhaber SZ, Sher DJ, D'Amico AV: Death from high-risk prostate cancer versus cardiovascular mortality with hormone therapy. Cancer 2013, 119:1808-1815.

2. Hoffman RM, Koyama T, Kang-Hsien F, Albertsen PC, Barry MJ, Goodman M, Hamilton AS, Potosky AL, Stanford JL, Stroup AM, Penson DF: Mortality after radical prostatectomy or external beam radiotherapy for localized prostate cancer. J Natl Cancer Inst 2013, 105:711-718.

3. Abdollah F, Sun M, Thuret $R$, Jeldres C, Tian Z, Briganti A, Shariat SF, Perrotte $P$, Rigatti P, Montorsi F, Karakiewicz PI: A competing-risks analysis of survival after alternative treatment modalities for prostate cancer patients: 1988-2006. Eur Urol 2011, 59:88-95

4. Abdollah F, Schmitges J, Sun M, Jeldres C, Tian Z, Briganti A, Shariat SF, Perrotte P, Montorsi F, Karakiewicz PI: Comparison of mortality outcomes after radical prostatectomy versus radiotherapy in patients with localized prostate cancer: a population-based analysis. Int J Urol 2012, 19:836-844.

5. Kibel AS, Ciezki JP, Klein EA, Reddy CA, Lubahn JD, Haslag-Minoff J, Deasy JO, Michalski JM, Kallogjeri D, Piccirillo JF, Rabah DM, Yu C, Kattan MW Stephenson AJ: Survival among men with clinically localized prostate cancer treated with radical prostatectomy or radiation therapy in the prostate specific antigen era. J Urol 2012, 187:1259-1265.

6. Koie T, Ohyama C, Yamamoto H, Hatakeyama S, Yoneyama T, Hashimoto Y, Kamimura N: Safety and effectiveness of neoadjuvant luteinizing hormone-releasing hormone agonist plus low-dose estramustine phosphate in high-risk prostate cancer: a prospective single-arm study. Prostate Cancer Prostatic Dis 2012, 15:397-401
7. Koie T, Yamamoto H, Hatakeyama S, Kudoh S, Yoneyama T, Hashimoto Y, Kamimura N, Ohyama C: Minimum incision endoscopic radical prostatectomy: clinical and oncological outcomes at a single institute. Eur J Surg Oncol 2011, 37:805-810.

8. Roach M III, Hanks G, Thames H Jr, Schellhammer P, Shipley WU, Sokol GH, Sandler H: Defining biochemical failure following radiotherapy with or without hormonal therapy in men with clinically localized prostate cancer: recommendations of the RTOG-ASTRO Phoenix consensus conference. Int J Radiat Oncol Biol Phys 2006, 65:965-974.

9. Rubin DB, Thomas N: Matching estimated propensity scores: relating theory to practice. Biometrics 1996, 52:249-264.

10. Edge SB, Byrd DR, Compton CC, Fritz AG, Greene FL, Trotti A (Eds): AJCC cancer staging manual. 7th edition. New York: Springer; 2010.

11. Epstein Jl, Allsbrook WC Jr, Amin MB, Egevad LL, ISUP Grading Committee: The 2005 International Society of Urological Pathology (ISUP) consensus conference on Gleason grading of prostatic carcinoma. Am J Surg Pathol 2005, 29:1228-1242

12. Grimm P, Billiet I, Bostwick D, Dicker AP, Frank S, Immerzeel J, Keyes M, Kupelian P, Lee WR, Machtens S, Mayadev J, Moran BJ, Merrick G, Millar J, Roach M, Stock R, Shinohara K, Scholz M, Weber E, Zietman A, Zelefsky M, Wong J, Wentworth S, Vera R, Langley S: Comparative analysis of prostatespecific antigen free survival outcomes for patients with low, intermediate and high risk prostate cancer treatment by radical therapy: results from the prostate cancer results study group. BJU Int 2012, 109:22-29.

13. Walz J, Joniau S, Chun F, Isbarn H, Jeldres C, Yossepowitch O, Chao-Yu H, Klein EA, Scardino PT, Reuther A, Poppel HV, Graefen M, Huland H, Karakiewics PI: Pathological results and rate of treatment failure in high-risk prostate cancer patients after radical prostatectomy. BJU Int 2010, 107:765-770.

14. Xylinas $E$, Daché $A$, Rouprêt $M$ : Is radical prostatectomy a viable therapeutic option in clinically locally advanced [T3] prostate cancer? BJU Int 2010, 106:1596-1600.

15. Soloway MS, Pareek K, Sharifi R, Wajsman Z, McLeod D, Wood DP Jr, Puras-Baez A, Lupron Depot Neoadjuvant Prostate Cancer Study Group: Neoadjuvant androgen ablation before radical prostatectomy in cT2bNxM0 prostate cancer: 5-year results. J Urol 2002, 167:112-116.

16. Pollack A, Zagars GK, Smith LG, Lee JJ, von Eschenbach AC, Antolak JA, Starkschall G, Rosen I: Preliminary results of a randomized radiotherapy dose-escalation study comparing $70 \mathrm{~Gy}$ with $78 \mathrm{~Gy}$ for prostate cancer. J Clin Oncol 2000, 18:3904-3911.

17. Bolla M, Gonsalez D, Warde P, Dubois JB, Mirimanoff RO, Storme G, Bernier J, Kuten A, Sternberg C, Gil T, Collette L, Pierart M: Improved survival in patients with locally advanced prostate cancer treated with radiotherapy and goserelin. N Eng J Med 1997, 337:295-300.

18. Lee WR, Hanlon AL, Hanks GE: Prostate specific antigen nadir following external beam radiation therapy for clinically localized prostate cancer: the relationship between nadir level and disease free survival. J Urol 1996, 156:450-453.

doi:10.1186/1477-7819-12-134

Cite this article as: Koie et al:: Both radical prostatectomy following treatment with neoadjuvant LHRH agonist and estramustine and radiotherapy following treatment with neoadjuvant hormonal therapy achieved favorable oncological outcome in high-risk prostate cancer: a propensity-score matching analysis. World Journal of Surgical Oncology 2014 12:134. 